\title{
Lifestyle medicine in community-engaged health promotion
}

\author{
Sherldine Tomlinson ${ }^{1,2}$ (D)
}

Received: 10 September 2019 / Accepted: 30 April 2020 / Published online: 29 May 2020

(C) Springer-Verlag GmbH Germany, part of Springer Nature 2020

\section{Global health crisis}

Globally, chronic diseases (CDs) or non-communicable conditions are soaring at an alarming rate. The number of cases is predicted to climb even more with ageing populations, globalisation, and urbanisation. CDs have disabled or taken the lives of many people of all age groups, disproportionately affecting people in low- and middle-income countries and populations (WHO 2018; Higuchi 2010; WHO 2005). The World Health Organisation (WHO) estimates that 14 million people each year die from a chronic disease, which is equivalent to $71 \%$ of all deaths worldwide (WHO 2018). Cardiovascular diseases account for most of the world's CD deaths, or 17.9 million people annually, followed by 9.0 million from cancers, 3.9 million from respiratory illnesses, and 1.6 million from diabetes (WHO 2018). The world chronic diseases figures are enormous, and they will continue to have an impact on people's lives, particularly for those living in developed countries as well as marginalised groups in lowincome neighbourhoods.

The 2017 total burden of non-communicable diseases by cause as expressed in age-standardised disability-adjusted life years (DALY) per 100,000 individuals at the global level has been estimated to be around 19,676.50 (Roser and Ritchie 2019). When looking at lower-income regions such as SubSaharan Africa, there is a shift of burden, where the CDs in the last two decades are mixed. The DALY increased from 90.6 million per 100,000 individual (95\% uncertainty interval 81 . $0-101.9)$ in 1990 to 151.3 million per 100,000 individual (133.4-171.8) in 2017 (Gouda 2019). In contrast, figures for DALY in higher-income countries like Canada and the United States declined. The decrease in DALY suggests that the

\section{Sherldine Tomlinson}

1 School of Biomedical Science, Faculty of Science, Charles Sturt University, Locked Bag 588, Wagga Wagga, NSW 2678, Australia

2 Present address: Toronto, Canada health of individuals in these countries is improving. Perhaps a contributing factor is the accessibility of public health promotion strategies to treat and prevent chronic diseases.

Analogous to the WHO data, premature death and morbidity for CDs in Canada are of concern. Statistics Canada in 2016 reported cancer and heart disease as the two leading causes of death, accounting for $48.6 \%$ of all mortality in the country (Statistics Canada 2016). The total deaths from cancer were 79,084 , while diseases of the heart were 69,576 (Statistics Canada 2016). The loss of life due to diabetes was 6838, while the death toll from cerebrovascular diseases (e.g., stroke) was 13,551 (Statistics Canada 2016). Other chronic conditions affecting some Canadians included high blood pressure/ hypertension (HTN), asthma, mood disorders, and musculoskeletal diseases (Statistics Canada 2020). The number of CDs in Canada and globally significantly shrink patients' lifespan, where it causes early death, and could lead to other adverse consequences.

The impact of chronic conditions is burdensome, causing a substantial fiscal burden on healthcare systems. In 2016, for instance, it was estimated that chronic diseases and other ailments cost the Canadian economy $\$ 190$ billion annually, with $\$ 122$ billion in indirect income and productivity losses (Kaczorowski et al. 2016). Unfortunately, the economic burden of diseases on healthcare systems in developed countries is more substantial, and therefore, it is challenging to meet the needs of chronic disease patients. A $2004 \mathrm{WHO}$ report predicted that in the next 50 years, the number of patients requiring care will increase twofold in low-income regions such as the Caribbean and Latin America, and triple in sub-Saharan Africa (Epping-Jordan et al. 2004). The growing threat will put more strain on the already fragile healthcare systems, and to some extent hinder the economic development of some regions, especially in low-income nations. It then makes sense that sustainable approaches to reducing the chances of people experiencing or managing CDs should be employed. Perhaps existing knowledge or a series of low-cost, high-impact action plans at the community level could be a starting point. Such strategies would not only reduce chronic illnesses but also remove healthcare gaps. 
A new strategic approach that is altering the preventative care landscape and is having a dramatic influence on preventing chronic disorders is lifestyle medicine (LM). Lifestyle medicine is defined as "a branch of evidencebased medicine in which comprehensive lifestyle changes (including nutrition, physical activity, stress management, social support, and environmental exposures) are used to prevent, treat, and reverse the progression of chronic disease, by addressing their underlying causes" (The Canadian Academy of Lifestyle Medicine n.d.). Evidence-based research suggests that LM recommendations could extend the lives of disease risk individuals allowing them to live longer with fewer disabilities. A long-term community-based combined exercise initiative, for example, was shown to improve patients' glycaemic control, lipid profile, blood pressure, and anthropometric profile (Mendes et al. 2017). Community lifestyle interventions to support proper dietary intake, as well as offering smoking cessation counselling, have also been beneficial to lower individuals exposure to lifestyle risk (Primary Care Review 2010; Dollahite et al. 2014). These community-engaged schemes are indications that LM is suitable for health improvement, mainly because its practices are integrated into the clinical model of care and slowing disease development, making its way to the community model of prevention. At the community level, LM is desirable to address the at-risk populations as well as other disadvantaged groups. The current paper looks at community-engaged lifestyle medicine to build healthier communities and health equity.

\section{Health and the community}

The definition of community can mean many different things, and thus, there is no absolute meaning to the word. Since the late nineteenth century, "the use of the term community has remained to some extent associated with the hope and the wish of reviving once more the closer, warmer, more harmonious type of bonds between people vaguely attributed to past ages" (Elias 1974, quoted by Hoggett 1997). Some have even focused on the community as a geographical area or space, while others have centred on groups of people living in a specific place or looking at the community as an area of ordinary life (Elias 1974, quoted by Hoggett 1997). Therefore, in a broader perspective, the community is space where people inhabit, where they live work, play, can interact in many different ways, and a place where they use resources of all kinds (Hoggett 1997). The definitions demonstrate the multiple ways communities are different as they relate to belonging, geographical space, and interaction. What is significantly missing is their uniqueness in terms of diversity of cultural differences, or by traditions.
Communities are robust when there are investments in social programmes and initiatives that strive to better the lives of its members (McMurray and Clendon 2015). Authors McMurray and Clendon (2015) cited that a central part of community building is "protecting the community from harm or stagnation, including helping its citizens to enhance their existing capacity for future development by fostering health literacy (in other words, providing community members with the knowledge and resources that contribute to their health and wellbeing) and working in partnership with them to become empowered to make decisions that will maintain the community's viability and capability to cope with any future challenges". Living in a healthy environment means that the health of the community is everyone's responsibility. In other words, an ecological approach is welcomed. As such, collective efforts are encouraged to help build a "culture of health." The Robert Wood Johnson Foundation first coined the term, and it referred to health seekers or community members having the opportunity to make healthier choices and engage in active and healthy lifestyles (i.e., exercise, recreational activities, nutrition programmes) (McCollum et al. 2017). For this to emerge, an unprecedented collaboration is required, where local governments, stakeholders, healthcare professionals, and others need first to recognise and understand the underlying causes, the social determinants of health (McMurray and Clendon 2015). Accepting the social factors that influence health outcomes improves health equity, which in turn builds healthier communities. Realistically, leadership from local governments with other stakeholders to address the determinants is the only way the culture of health can exist.

As indicated, encouraging a healthy culture in communities takes a multifaceted approach. Some coordinated efforts could involve gaining knowledge about health promotion, applying evidence-based research on interventions, and acquiring a strong commitment to social engagement within the community. These measures can help to foster the intersectional relationships, and build professional collaborations and continuous communication among the various stakeholders (McMurray and Clendon 2015; Navarro et al. 2007). Equally, systematic assessment skills, as well as constant surveillance and monitoring, implementing evaluation strategies, community advocacy, community development ,and cost-effective interventions through financial investments, are obligatory to attain a healthy culture (McMurray and Clendon 2015; Navarro et al. 2007). Partnering with all sources is desirable to build community health capacity, thus benefiting all neighbourhoods. Building a healthy community increases the usefulness and quality of health promotions, which allows all members the opportunity to live a healthier life. 


\section{Lifestyle medicine in community-engaged health promotion}

The concept of LM first originated as a component of physicians' internal medical practice and is an adjunct to traditional medicine. Physicians apply the training as a strategy to coach health seekers to be more active in their care (Sagner et al. 2014; Polak et al. 2015; Egger et al. 2009; Dacey et al. 2013). Physician LM coaching involves health risk assessment, health behaviour change counselling, and clinical application of lifestyle modification (Sagner et al. 2014). The approach was first introduced in American postgraduate medical training courses to educate medical students about exercise prescription and nutrition (Polak et al. 2015; Egger et al. 2009). In a 1975 survey, it was found that only $16 \%$ of medical schools across the United States offered courses that focused on exercise (Polak et al. 2015). Another survey in 1985 indicated that just $27 \%$ of medical schools provided the required $25 \mathrm{~h}$ of nutrition education, whereas more than $50 \%$ did not offer courses addressing exercise prescription (Polak et al. 2015). One more questionnaire found that $94 \%$ of medical school students perceived the competence to recommend exercise to patients as either 'moderately important' or 'important,' with only $10 \%$ of graduates felt they were proficient in prescribing exercise regimens (Polak et al. 2015). The lack of training in exercise prescription or nutrition counselling suggested the requirement for change, and subsequently, this has prompted the action of leaders across medical professions to integrate lifestyle education into medical school curricula (Sagner et al. 2014; Polak et al. 2015). Medical students now have the opportunity to improve on their lifestyle counselling skills, equipping them with lifestyle information they can communicate to future patients as well for their health behaviours.

In recent years, LM has extended across the healthcare spectrum, where other clinicians and specialists (e.g., nurses, allied health workers, holistic practitioners, community health workers, dietitians) have fused its principles into their practice (Dacey et al. 2013). Indeed, this is good creativity, as health seekers can access lifestyle counselling and healthy activities other than in a physician's office. Related LM services have long been welcomed in community-focused health promotions such as health and wellness or health promotion. A multitude of health services is available in these environments, including primary and mental health care (e.g., emotional wellbeing/ stress management), nutrition counselling, and exercise and physical activity promotion providing support for health seekers (Dacey et al. 2013). On the organisational level, stakeholders invest in community health organisations and promotional activities, which makes them free of charge, creating opportunities for community members and health seekers to take care of their health.
Community health centres (CHCs) across Canada are offering residents primary care through a collaborative team approach, with services provided at no cost. CHCs integrate health promotion, prevention activities, and social services that are easily accessible to community members (Canadian Association of Community Health Centres 2016). LM in these clinics is a good fit to meet the needs of community residents, given that they already provide health care in addition to health prevention activities. A positive aspect of CHCs is they actively address the social determinant of health, where they support the removal of health disparities and barriers.

Faith-based health promotions such as the African American church have long combined elements of the LM principles in its health curricula. With no-cost peer-led lifestyle interventions, the African American church provides churchgoers and community members with diabetes, blood pressure, and weight management services, breast cancer screening, and smoking cessation sessions (Tomlinson 2011; Pattillo-McCoy 1998). Historically, the Black church long served as pillars of community leadership and sites of social interaction in Black communities (Tomlinson 2011; PattilloMcCoy 1998). It is highly respected, and the clergy within these churches are trusted gatekeepers to provide health education counselling and to encourage healthy behaviour change (Tomlinson 2011). Most notably, the delivery of church-based lifestyles aimed at African Americans integrates cultural elements, which makes them attractive and practical to attend (Dacey et al. 2013).

Established in the fall of 2016, the Rexdale Women's Centre (RWC), located in Toronto, ON, Canada introduced a new health and wellness initiative aimed at newcomers and permanent residents. The scheme was offered to empower and support these clients' health and to reduce barriers. The programme addresses the social determinant of health; it follows the principles of community-based participatory research, and applies evidence-based research, with added cultural appropriateness. Weekly healthy living workshops, fitness classes, community healthy living events, and one-on-one health support are components of the programme. To achieve a better understanding of what the issues are which surround the client's mental health and other health concerns, the programme's coordinator included referrals, thus partnering with other community health agencies. Thus far, RWC's health and wellness programme continues to thrive as reported on the programme's satisfaction surveys and client's feedback.

With the availability of community health services, LM is a means for members, including marginalised and other vulnerable groups, to achieve full potential in life. Too often, these populations do not get the chance to participate in healthpromoting activities because of their socioeconomic conditions or other reasons. Unfortunately, the inability to get them involved in healthy lifestyles causes further health difficulties. 
Low-income communities have a higher prevalence of CDs and are susceptible to lifestyle risk factors (Allen et al. 2017). As a consequent of their poor health outcome, these groups experience lower life expectancy than the average population. Community LM-related health promotion initiatives and services have proven useful for disadvantaged groups. Often, societal factors in understanding the health behaviours of these populations are complex and loaded because of the many factors involved. Even so, this activity opens the door for discussions and innovated solutions to fill the disparity gap, making it possible for all health seekers to achieve equitable access to health promotion activities.

It should be emphasised that if community LM is to have an active role in reducing health inequality and improving the health of people, victim-blaming should be avoided (Krishnaswami et al. 2018). Often blaming individuals for their unhealthy behaviours can occur, especially those having lower socioeconomic status. The evidence suggested that supporting all people to lead a healthier life is the primary goal.

\section{Conclusion}

Most CDs are preventable and are mainly the result of unhealthy practices (Madaki and Danjuma 2014; Bodai et al. 2018). Adopting healthy lifestyle choices can significantly lower the risk of getting a chronic condition. However, it is not as easy as suggested. Most people want to live a healthy life, but they do not know where to start or how to follow a living lifestyle regiment. It does not help that health seekers receive little information from primary care professionals, most notable their family physicians on how to implement long-term plans to achieve healthful living (Barnes and Schoenborn 2012; Kolasa and Rickett 2010). The lack of knowledge is a challenge, and as such, embracing the importance of living a healthy lifestyle in health is critical.

Furthermore, the provision of LM-related services in community health makes much sense because it offers health seekers more than just one-on-one consultation on a healthy lifestyle. It provides them with opportunities to participate in health services (i.e., health risk assessment, management of chronic conditions, health action plans, health literacy, group fitness classes, and healthy living workshops, and more). Selfmanagement support in community LM also assists health seekers with setting goals, empowerment thus helping to boost their self-efficacy. A significant impact is that the multiplicity of healthy activities provided in community health settings could have a more lasting influence than just the individual system with physicians. There is team support and the use of community resources (Primary Care Review 2010). From an LM perspective, community-engaged health is critical and valuable in all aspects to treat, prevent, and manage increasing community members' activation. The practice requires a comprehensive approach to community engagement, and as such collective action by all organisational levels is a must. This does not only support community-engaged programming, but it also helps to extend LM activities, therefore adding creditability to the practice.

Future research to explore specific pathways and processes by which LM can be delivered in community settings, as well as the theoretical and practical implications to promote healthy living, should be welcomed.

\section{Compliance with ethical standards}

Conflict of interest statement None

Ethical approval None

Informed consent [optional] None

\section{References}

Allen L et al (2017) Socioeconomic status and non-communicable disease behavioural risk factors in low-income and lower-middleincome countries: a systematic review. Lancet Glob Health 5(3): e277-e289. https://doi.org/10.1016/S2214-109X(17)30058-X

Barnes PM, Schoenborn CA (2012) Trends in adults receiving a recommendation for exercise or other physical activity from a physician or other health professional. NCHS Data Brief 86:1-8. https://www. cdc.gov/nchs/data/databriefs/db86.pdf

Bodai BI, Nakata TE, Wong WT, Clark DR et al (2018) Lifestyle medicine: a brief review of its dramatic impact on health and survival. Perm J 22:17-025 https://www.ncbi.nlm.nih.gov/pmc/articles/ PMC5638636/

Canadian Association of Community Health Centres (2016) Community health centres: evidence \& impact. Canadian Association of Community Health Centres, Toronto. https://www.cachc.ca/chcsevidence-impact/

Dacey M, Arnstein F, Kennedy MA et al (2013) The impact of lifestyle medicine continuing education on provider knowledge, attitudes, and counseling behaviors. Med Teach 35(5):e1149-e1156. https:// www.tandfonline.com/doi/pdf/10.3109/0142159X.2012.733459

Dollahite JS, Pijai EI, Scott-Pierce M, Parker C, Trochim WJ (2014) A randomized controlled trial of a community-based nutrition education program for low-income parents. Nutr Educ Behav 46(2):102109. https://doi.org/10.1016/j.jneb.2013.09.004

Egger GJ, Binns AF, Rossner SR (2009) The emergence of "lifestyle medicine" as a structured approach for management of chronic disease. Med J Aust 190(3):143-145. https://doi.org/10.5694/j.13265377.2009.tb02317.x

Epping-Jordan JE, Pruitt SD, Bengoa R, Wagner EH (2004) Improving the quality of health care for chronic conditions. Qual Saf Health Care 13(4):299-305. https://qualitysafety.bmj.com/content/qhe/13/ 4/299.full.pdf

Gouda HN (2019) Burden of non-communicable diseases in sub-Saharan Africa, 1990-2017: results from the Global Burden of Disease Study 2017. Lancet Glob Health 7(10):e1375-e1387. https://doi.org/10. 1016/S2214-109X(19)30374-2

Hoggett P (1997) Contested communities. In: Hoggett P (ed) Contested communities. Experiences, struggles, policies. Policy Press, Bristol. Online document 
Kaczorowski J, Campbell NR, Duhaney T et al (2016) Reducing deaths by diet: call to action for a public policy agenda for chronic disease prevention. Can Fam Physician 62(6):469-470

Kolasa KM, Rickett K (2010) Barriers to providing nutrition counseling cited by physicians: a survey of primary care practitioners. Nutr Clin Pract Oct 25(5):502-509. Accessed May 2019. https://doi.org/10. $1177 / 0884533610380057$

Krishnaswami J, Jaini PA, Howard R, Ghaddar S (2018) Communityengaged lifestyle medicine: building health equity through preventive medicine residency training. Am J Prev Med 55(3):412-421. https://doi.org/10.1016/j.amepre.2018.04.012

Primary Care Review (2010) Lifestyle medicine: an overview. Prim Care Rep May 2010. Accessed Nov 2019. https://www.reliasmedia.com/ articles/19034-lifestyle-medicine-an-overview

Madaki JK, Danjuma SA (2014) Lifestyle medicine: a crucial strategy for optimal health and longevity. JJM 8(1):23-29. Accessed May 2019. https://www.ajol.info/index.php/jjm/article/view/105954/95945

McCollum M, Kovner C, Ojemeni MT et al (2017) Nurses improve their communities' health where they live, learn, work, and play. Policy Polit Nurs Pract 18(1):7-16 Accessed http://journals.sagepub.com/ doi/full/10.1177/1527154417698142\# i25

McMurray A, Clendon J (2015) Community health and wellness-e-book: Primary health care in practice. Elsevier Health Sciences. https:// scholar.google.com/scholar lookup?hl=en\&publication year= 2011\&author $=$ A. + McMurray \&author $=$ J.+Clendon\&title $=$ Community+Health+and+Wellness $\% 3 \mathrm{~A}+$ Primary+Health+Care+ in+Practice

Mendes R, Sousa N, Resi VM, Themudo-Barata JL (2017) Implementing low-cost, community-based exercise programs for middle-aged and older patients with type 2 diabetes: what are the benefits for glycemic control and cardiovascular risk? Int J Environ Res Public Health 14(9):1057. https://doi.org/10.3390/ijerph14091057

Navarro AM, Voetsch KP, Liburd et al (2007) Charting the future of community health promotion: recommendations from the National Expert Panel on Community Health Promotion. Prev Chronic Dis 4(3):A68-A68

Pattillo-McCoy M (1998) Church culture as a strategy of action in the black community. Am Sociol Rev 767-784. https://www.scholars. northwestern.edu/en/publications/church-culture-as-a-strategy-ofaction-in-the-black-community

Polak R, Pojednic RM, Phillips EM (2015) Lifestyle medicine education. Am J Lifestyle Med 9(5):361-367

Roser M, Ritchie H (2019) "Burden of Disease". OurWorldInData.org. Assessed Nov 2019. https://ourworldindata.org/burden-of-disease' [Online Resource]

Sagner M, Katz D, Egger G, Lianov L, Schulz KH, Braman M, Ornish D (2014) Lifestyle medicine potential for reversing a world of chronic disease epidemics: from cell to community. Int J Clin Pract 68(11): $1289-1292$

Statistics Canada (2016) Death, causes of death and life expectancy. Statistics Canada, Ottawa. https://www150.statcan.gc.ca/n1/dailyquotidien/180628/dq180628b-eng.htm

Statistics Canada (2020) Table 13-10-0394-01 Leading causes of death, total population, by age group. Statistics Canada, Ottawa. https:// www150.statcan.gc.ca/t1/tbl1/en/tv.action?pid=1310039401

The Canadian Academy of Lifestyle Medicine (n.d.) Accessed https:// www.calmlifestylemedicine.ca/lifestyle-medicine/what-is-lifestylemedicine

Tomlinson S (2011) Evidence to support church-based health promotion programmes for African Canadians at risk for cardiovascular disease. J Immigr Minor Health 13(6):1175. https://doi.org/10.1007/ s10903-011-9502-5

Higuchi M (2010) Lifestyle Diseases: Access to Chronic Disease Care in Low- and Middle-Income Countries. United Nations, New York. https:/unchronicle.un.org/article/lifestyle-diseases-access-chronicdisease-care-low-and-middle-income-countries

WHO (2018) Noncommunicable diseases fact sheets. World Health Organisation, Geneva. http://www.who.int/news-room/fact-sheets/ detail/noncommunicable-diseases. Accessed 10 September 2019

World Health Organization (2005) Chronic diseases in low- and middleincome countries. World Health Organisation, Geneva. http://www. who.int/chp/chronic_disease_report/media/Factsheet3.pdf. Accessed 10 September 2019

Publisher's note Springer Nature remains neutral with regard to jurisdictional claims in published maps and institutional affiliations. 